\title{
Burdens of proof of misconduct
}

Despite well publicized cases of scientific fraud, and less publicized examples of other misbehaviour by researchers, calls for enhanced external policing of science need to be greeted sceptically.

LAST week was a stimulating one for those who believe that the scientific process needs external policing. Martin Fleischmann and Stanley Pons, of "cold fusion" fame, lost a court case in Italy in which they had attempted to sue a journalist who linked their work too closely for their liking to with the concept of fraud (see page 369). A Brazilian virologist was successfully sued to the tune of $\$ 50,000$ for plagiarism of unpublished research (see page 371). And in the United Kingdom the British Medical Journal and The Lancet, acting in a combined initiative, called for the establishment of a central agency to tackle misconduct in research.

Nothing much is likely to happen fast in consequence of any of these. Most countries have been slow to follow the lead taken by the United States in establishing institutions to tackle this area. It is not surprising that the most vigorous proponents of such changes have come from medical research, given the potential dangers to patients. Such was the case in Denmark, the European leader in pursuing misconduct, whose medical research council played a key role in establishing the national Danish Committee on Scientific Dishonesty. This body includes scientists and lawyers nominated by interested parties that include, commendably, local political authorities. Other Scandinavian countries are actively pursuing similar possibilities, as is Australia. But Germany, France, Italy and the UK are prominent in their reluctance to respond to concerns in such an institutionalized fashion, to say nothing of Japan.

Is such reluctance advisable? That depends on whether those few cases of misconduct that are reported in such countries are merely the tip of an iceberg, or prove that, by and large, the science community's procedures - a combination of peer review, replication of results, employers' discipline and, occasionally, whistleblowing - are an effective form of self regulation. Certainly the factors such as citation records used increasingly to judge researchers' worth strengthen the pressure on individuals to behave badly - to bias their interpretation of data, or take too much credit for themselves. But evidence for an iceberg remains slender and anecdotal. That is one reason why calls for new agencies are unlikely to make much headway.

Significant expense is another. That is an obstacle which the United States Commission on Research Integrity (which advises Congress and the Department of Health and Human Services) may find insuperable. In its "Ryan report" produced late last year, the commission attracted the wrath of learned societies by proposing a range of initiatives that would impose additional costs on both the government and employers (as well as the societies). These included enhanced training and support for conferences in research ethics, and rigorously established procedures to deal with complaints, backed by quality control implemented with on-site visits by the Office of Research Integrity. (The report did not include an estimate of the costs.)

The critics are right to suggest that the Ryan report does not adequately make its case for such investment. But they would do well to express stronger concerns over the varied performance of institutions in carrying out their responsibilities, as reported by the commission. There is every reason to explore possible weaknesses in self regulation, in order to maintain public and political confidence in what is after all a costly public good.
The role of whistleblowers in particular can be critical. But it is also haphazard, and so, too, are the consequences of their actions, as evidenced by a recent ORI survey that documented a sizeable number of whistleblowers who experienced negative consequences. The Ryan report deserves support for promoting ways in which whistleblowers' interests can be more strongly protected.

European countries are right, given the apparently small scale of the problem, to be sceptical of the need for national agencies to address misconduct. But their institutions have tended to display too little energy in ensuring adequate self-policing. Stronger scrutiny, including systematic and independent investigation of researchers' experience of scientific bias and misrepresentation, is surely due.

\section{Avoid flaming rows}

The future of the Internet as a research tool looks healthy, but there are dangers in excessively rapid communication.

"SPEAK in anger, and you will make the best speech that you'll ever regret", is a well-known maxim, as is one cure for curtailing this capacity - "count to ten". Many Internet users are now realizing that the same rules apply to comportment in cyberspace. The need to think twice when using the Internet has little to do with the predictable potholes of participating in 'flaming' (insulting other users) or the use of capitals in e-mail, which in Internet etiquette equates with SHOUTING. Rather, it has to do with the new problems created both by the speed with which information can be transmitted over the Internet or put on a Web page, and the mutation in mentality that some individuals undergo when they get behind the wheel of their computer.

This week's Briefing (pages 377-381) takes a serious look at the Internet and where it is going. But along the way Nature assimilated some anecdotal evidence of disadvantages dogging the medium (some not a million miles from home). Some individuals who would usually refrain from attacking colleagues seem to lose all sense of restraint when behind the screen. Combine this Jekyll-and-Hyde transformation with the immediacy and telegraphic style of e-mail and you have the makings of diplomatic mayhem.

The speed with which data can be put on the Web has also led to cases where it has had to be retracted because it had not been analysed properly. Similarly, researchers have hastily posted data on the Web before its commercial potential had been considered. Scientists are old hands at shooting themselves in the foot by publishing before patenting. The Web has made the trigger even itchier.

The lesson is that e-mails and Web submissions should be left to cool in a drawer (or its electronic equivalent, the draft folder) for a duration that is directly proportional to their scientific importance or potential for controversy. And even though its deployment can seem nauseatingly twee, scientific manners could only benefit from greater use of :-). 